\title{
AEROSITE: Autonomous Environmental and Scientific SWGO site Characterization Instrument
}

\section{Ladislav Chytka, ${ }^{a, *}$ Dušan Mandát, ${ }^{a}$ Miroslav Pech, ${ }^{a}$ Daniel Staník, ${ }^{a}$ Jakub Vícha, ${ }^{a}$ Petr Trávníček, ${ }^{a}$ Martina Boháčová, ${ }^{a}$ Petr Tobiška, ${ }^{a}$ Tomasz Bulik, ${ }^{b}$ Marek Cieślar ${ }^{b}$ and Mariusz Suchenek ${ }^{b}$ on behalf of the SWGO Collaboration}

(a complete list of authors can be found at the end of the proceedings)

${ }^{a}$ FZU - Institute of Physics of the Czech Academy of Sciences, Czech Republic, Na Slovance 1999/2, Praha, Czech Republic

${ }^{b}$ Astronomical Observatory, University of Warsaw, Aleje Ujazdowskie 4, 00478 Warsaw, Poland

E-mail: ladislav.chytka@fzu.cz

The project Southern Wide-field Gamma-ray Observatory (SWGO) aims to build an array of airshower detectors in the Southern hemisphere. Preliminary site searches identified suitable sites in Argentina, Bolivia, Chile and Peru. Site environment (including weather, seismic activity and also the electric field) is one of the key aspects to be considered in the site selection and should be based on reliable and comparable measurements.

We describe an environmental monitoring device to equip several candidate sites proposed for the SWGO. The individual monitoring sensors, control unit and the data storage together with the power system and data transfer concepts are specified. We also present the results of a long term cross-calibration campaign and a climate chamber evaluation of the proposed devices.

$37^{\text {th }}$ International Cosmic Ray Conference (ICRC 2021)

July 12th-23rd, 2021

Online - Berlin, Germany

\footnotetext{
*Presenter
} 


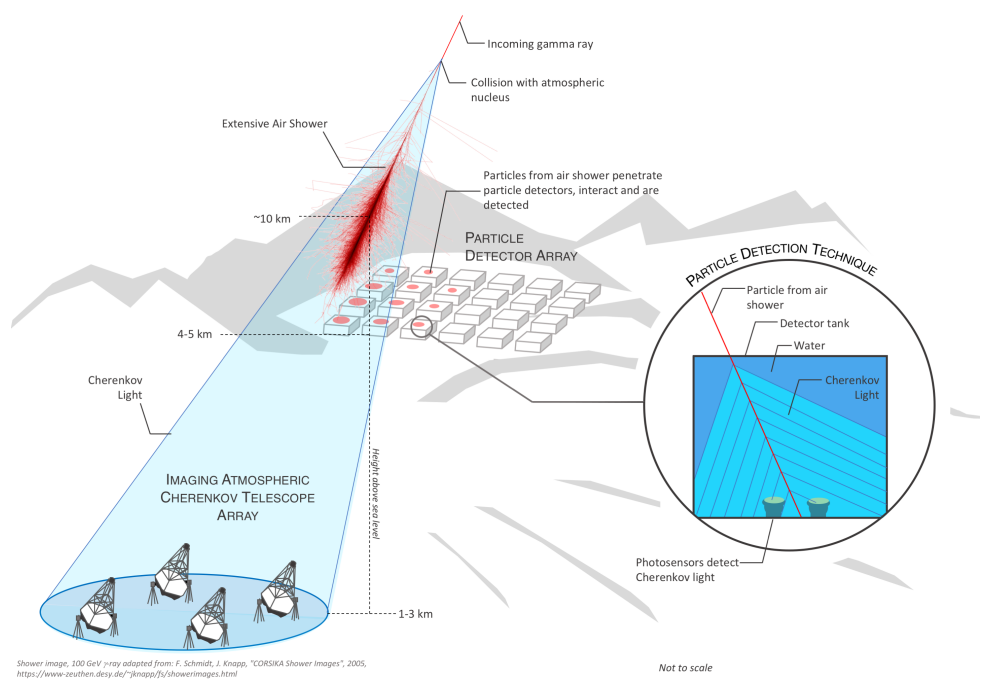

Figure 1: Gamma-ray detection illustration (courtesy of SWGO).

\section{Introduction}

The Southern Wide-field Gamma-ray Observatory (SWGO $)^{1}$ is a project of a future gamma-ray observatory to be built in the Southern Hemisphere. SWGO aims for being installed at a minimum altitude of $4.4 \mathrm{kmas} 1$ in order to reach a low energy range, down to hundreds of $\mathrm{GeV}$, in addition to detecting gamma-rays all the way up to several hundreds of TeV. It will complement Northern Hemisphere observatories like HAWC [1], ARGO [2] or LHASSO [3], utilizing an array of water Cherenkov detectors arranged in a high fill factor core detector with area considerably larger than HAWC and significantly better sensitivity, and low density outer array. [4]. An illustration of SWGO gamma-ray measurement is shown in Fig. 1. Candidate sites are located in South America: Argentina, Bolivia, Chile and Peru.

SWGO is currently in an R\&D phase - science benchmark cases are being defined, detector design and optimal site are being selected, simulation and analysis tools are being developed. One of the key aspects of optimal site selection is a detailed characterization of pre-selected sites. We present an instrument for a medium term site evaluation - Autonomous EnviROnmental and Scientific SWGO site characterization InsTrumEnt (AEROSITE).

\section{AEROSITE instrument}

AEROSITE is an off-grid environmental monitoring device to be deployed on four SWGO candidate sites to provide on-site measurements of temperature, humidity, atmospheric pressure, solar irradiation, wind speed and direction, electric field (E field) intensity and seismic activity.

The mechanical setup of the station consists of a steel IP 65 box to accommodate and protect a battery, charger and electronics. The weight of the battery and the box itself ( $\approx 90 \mathrm{~kg}$ total) ensures the stability of the station position. The box is fixed to a support frame to increase the distance between the ground and protect the electronics against a water leaking in case of heavy

\footnotetext{
${ }^{1}$ http://www.swgo.org
} 


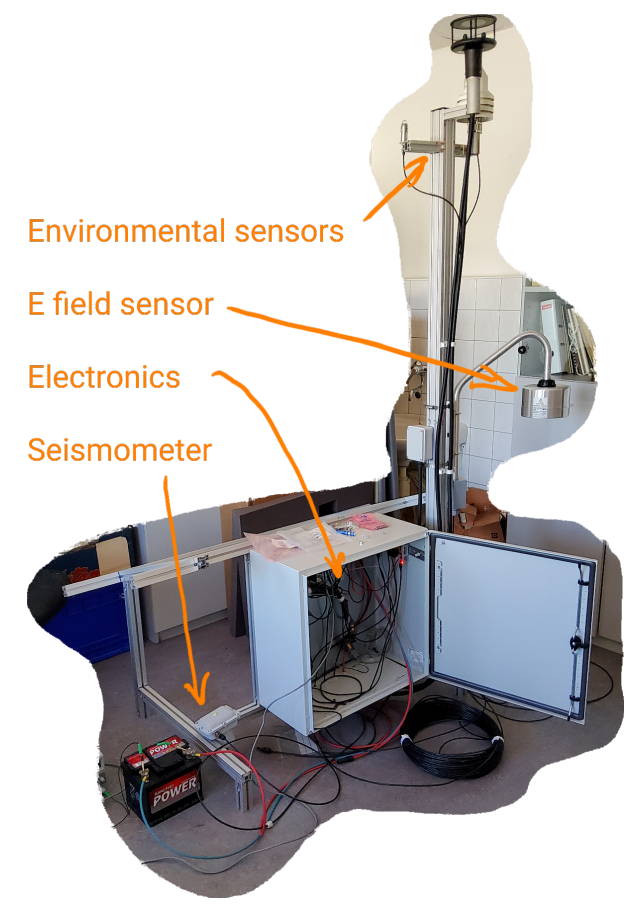

Figure 2: AEROSITE assembly.

rain. The support frame is made of Bosch type aluminum profiles. The profiles are rigid, light, easily adaptable and easy to assemble without welding. Weather and wind sensors are installed on a rigid mast with $2 \mathrm{~m}$ height. The sensor location is selected such that there is no shadowing of the wind or irradiation sensor. In default configuration, there is a $150 \mathrm{Whp}$ solar panel attached to the frame providing power for the entire station. A photograph of the AEROSITE assembly is in Fig. 2

Barometric pressure, humidity and temperature is measured by Reinhardt DFT 55V. The temperature range is $-40{ }^{\circ} \mathrm{C}$ to $60{ }^{\circ} \mathrm{C}$ with $0.01{ }^{\circ} \mathrm{C}$ resolution and $0.3{ }^{\circ} \mathrm{C}$ accuracy. The relative humidity range is $10 \%$ to $100 \%$ with $0.03 \%$ resolution and $\pm 2 \%$ accuracy. The range of the barometric pressure is $300 \mathrm{hPa}$ to $1200 \mathrm{hPa}$ with $0.01 \mathrm{hPa}$ resolution and $\pm 0.8 \mathrm{hPa}$ accuracy. The product from the German company Reinhardt [5] is very reliable and precise. The weather station was used within the ATMOSCOPE instrument during the CTA Site Selection Campaign [6].

Gill WindSonic [7] is proposed as the anemometer. It is a 2D ultrasonic anemometer with measurement range of the wind speed $0 \mathrm{~m} \mathrm{~s}^{-1}$ to $60 \mathrm{~m} \mathrm{~s}^{-1}$ with resolution of $0.01 \mathrm{~m} \mathrm{~s}^{-1}$ and accuracy of $\pm 2 \%$. The wind direction measurement ranges from $0^{\circ}$ to $359^{\circ}$, with resolution of $1^{\circ}$ and accuracy of $\pm 3^{\circ}$.

The solar irradiation is an important input into thermal simulations of water Cherenkov detectors and is affected by the local cloudiness. The sensor from Reinhardt company was selected and allows to measure the light of wavelengths in the range from $300 \mathrm{~nm}$ to $2800 \mathrm{~nm}$. The measured range of solar irradiation is $0 \mathrm{~W} / \mathrm{m}^{2}$ to $1500 \mathrm{~W} / \mathrm{m}^{2}$ with $0.3 \mathrm{~W}$ resolution and $\pm 40 \mathrm{~W}$ precision.

Strong electric fields can adversly affect measurement of water Cherenkov detectors, as observed by HAWC coleagues, hence E field is measured by Boltek EFM-100C [8] electric field mill with measurement range of $-20 \mathrm{kV} / \mathrm{m}$ to $20 \mathrm{kV} / \mathrm{m}$ and $5 \%$ accuracy.

Seismic activity measurement is provided by a seismometer designed by the group of T. Bulik 
from Polish Academy of Sciences [9].

Additionaly, AEROSITE can be equipped by a remote string of 14 temperature sensors (DS18B20, 2 of-the-shelf waterproof, 12 epoxy-potted) to monitor temperatures inside a prototype water tanks. These readings will be used for validation of thermal modeling of tanks.

Data acquisition of all sensors except for the seismometer is handled by Balena Fin computer [10], an industrial standard single board computer utilizing Raspberry Pi 3 compute module and equipped with $16 \mathrm{~GB}$ eMMC memory.

\section{Site characterization}

Site candidates were proposed by interested institutions meeting the initial criteria:

- altitude of $4.4 \mathrm{~km}$ or higher,

- location in South America at a latitude between 10 and 30 degrees south,

- water accessibility.

The following locations are being considered by SWGO as candidate observatory sites:

- Argentina: Cerro Vecar, Alto Tocomar (closeby)

- Bolivia: ALPACA site (Chacaltaya plateau)

- Chile: Pampa la Bola, Pajonales (closeby)

- Peru: Imata, lake Sibinacocha

The underlined sites are to be equipped by AEROSITE, as selected by the local site representatives. Argentinian and Bolivian sites have internet access and the AEROSITE data can be directly uploaded to our server. In Chile and Peru, periodic site visits (ca. every two months) are necessary to collect and upload the data to the server.

The site selection process is quite complex and environmental data are only one part of it. The environment is to be consider from the point of view of location (altitude, latitude), weather (storms, lightning, sun irradiance, freezing days, day/night temperature variation) and geology (rocks, terrain slope, earthquakes). Water source is crucial for water Cherenkov detectors, hence the distance, quality, supply rate, price of transport and possible environmental effect need to be considered. Other key variable is infrastructure, e.g. the power lines or internet connectivity. Also other site merits need to be evaluated ranging from site accessibility to political and administrative aspects (site ownership, taxes, import of equipment, local research institutes and companies support, labour rules, etc.). Details of site selection process are yet to be fixed.

Candidate sites environment will be evaluated based on:

- Public data - long term historical data obtained from nearby observatories, meteostations, satelites etc.

- AEROSITE data - data from cross-calibrated instruments to provide a reliable reference.

The public data come from various sources with different uncertainty and possible offsets and are mostly collected by instruments located rather far from the candidate sites. Hence, the AEROSITEs provide a way to calibrate these data and calculate reliable uncertainties. 


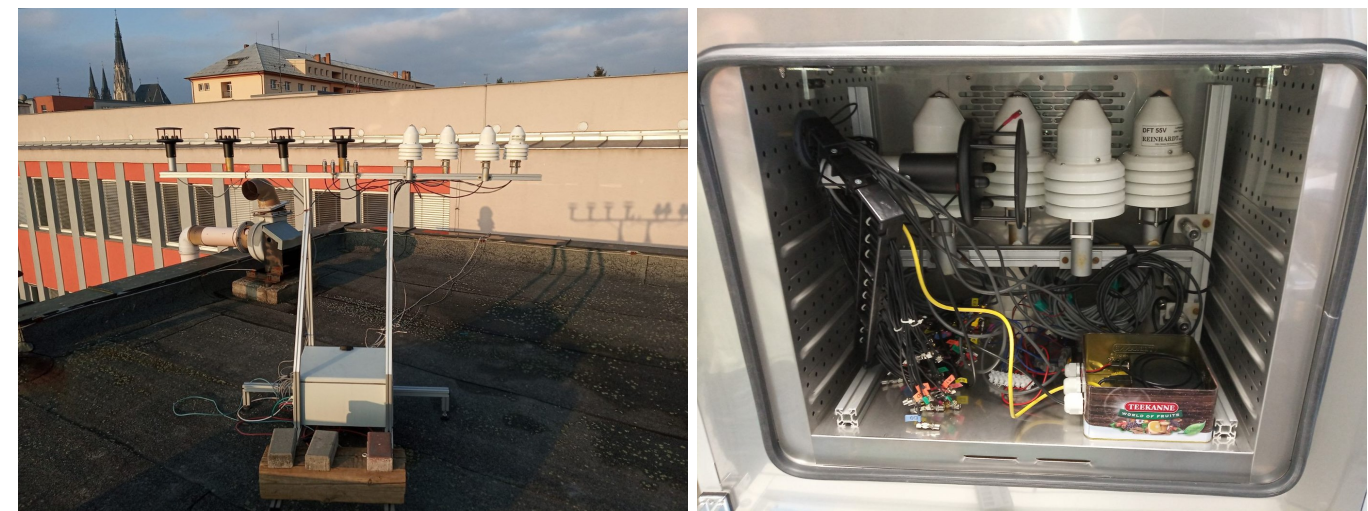

Figure 3: AEROSITE test campaign on JLO roof (left) and in the climate chamber (right).

\section{AEROSITE cross-calibration}

Weather sensors (DFT 55V, anemometers and irradiation sensors) were tested during a field test on the roof of Joint Laboratory of Optics (JLO) and in a climate chamber in order to obtain comparison of the four sets of the sensors and test stability of measurements. Figure 3 shows photographs of the two test setups.

\subsection{Field cross-calibration}

Four sets of environmental sensors were installed on roof of JLO from Oct 2020. There was about two weeks long break for the climate chamber test in November. Sets of sensors were removed between Jan and Apr 2021 for AEROSITEs assembly, hence between 2.5 and 5 months of data were collected for individual sets of sensors. Results from the first period are showin in Fig. 4.

There is a good agreement between the measurements of temperature, pressure and solar irradiance. Humidity measurement of set 1 manifests larger upward deviation (of about $5 \%$ ) after about two weeks of measurement. This was observed also in the second measurement period after the climate chamber tests, after which the set 1 was again reporting compatible humidity values.

The anemometer measurements are affected by the test setup placement, as it was on a roof with taller buildings surrounding it. All the anemometers were working during the both test periods and filtering the data using a two hour moving average shows reasonable agreement between all the sets, as shown in Fig. 5.

\subsection{Climate chamber test}

The four DFT $55 \mathrm{~V}$ sensors along with one anemometer were tested in a climate chamber in order to test behaviour under extended temperature and humidity ranges. The lowest temperature was set to $-20^{\circ} \mathrm{C}$ based on the lowest temperatures obtained from available data near the candidate sites. The high temperature of $35^{\circ} \mathrm{C}$ allowed low humidity measurements in the climate chamber.

The following tests were run:

- Temperature cycling $-20^{\circ} \mathrm{C}$ to $35^{\circ} \mathrm{C}$

- Temperature steps $-20,0,20$ and $35^{\circ} \mathrm{C}$ 

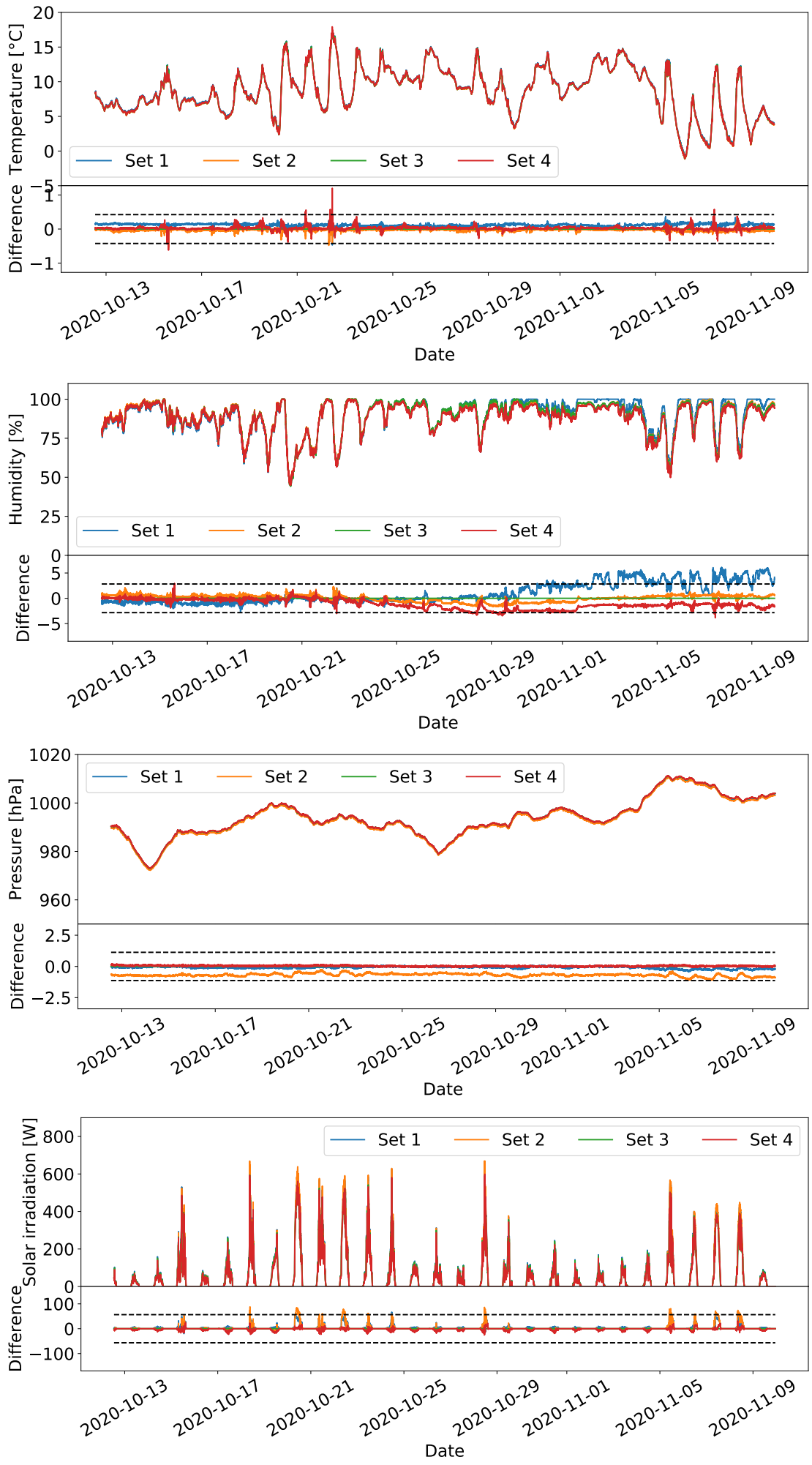

Figure 4: Results of field test campaign. Black dashed lines in difference plots mark declared accuracy. 


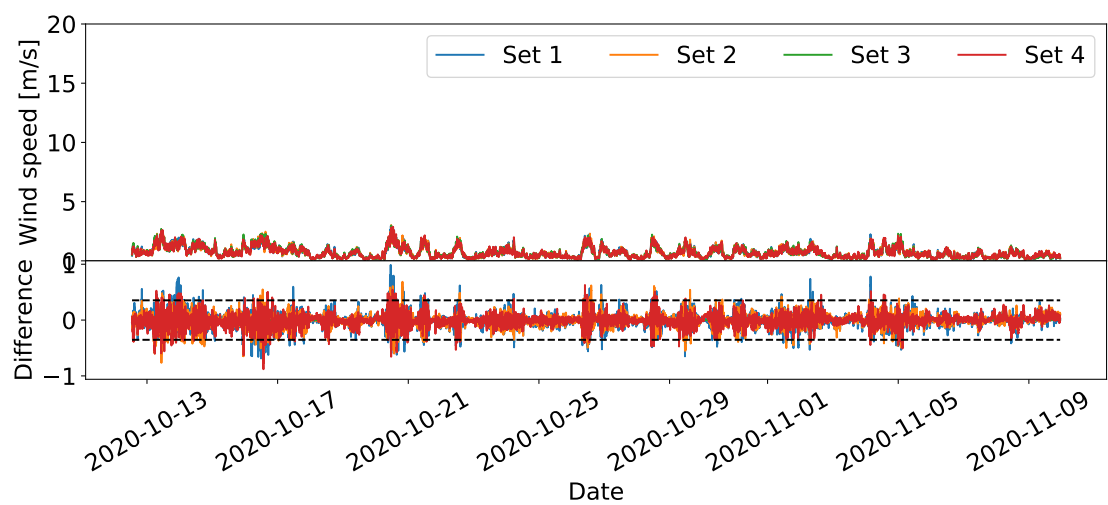

Figure 5: Results of field test campaign for the wind speed. Two hour moving average is applied on the data to filter random gusts due to neighbouring buldings. Black dashed lines in difference plots mark declared accuracy.
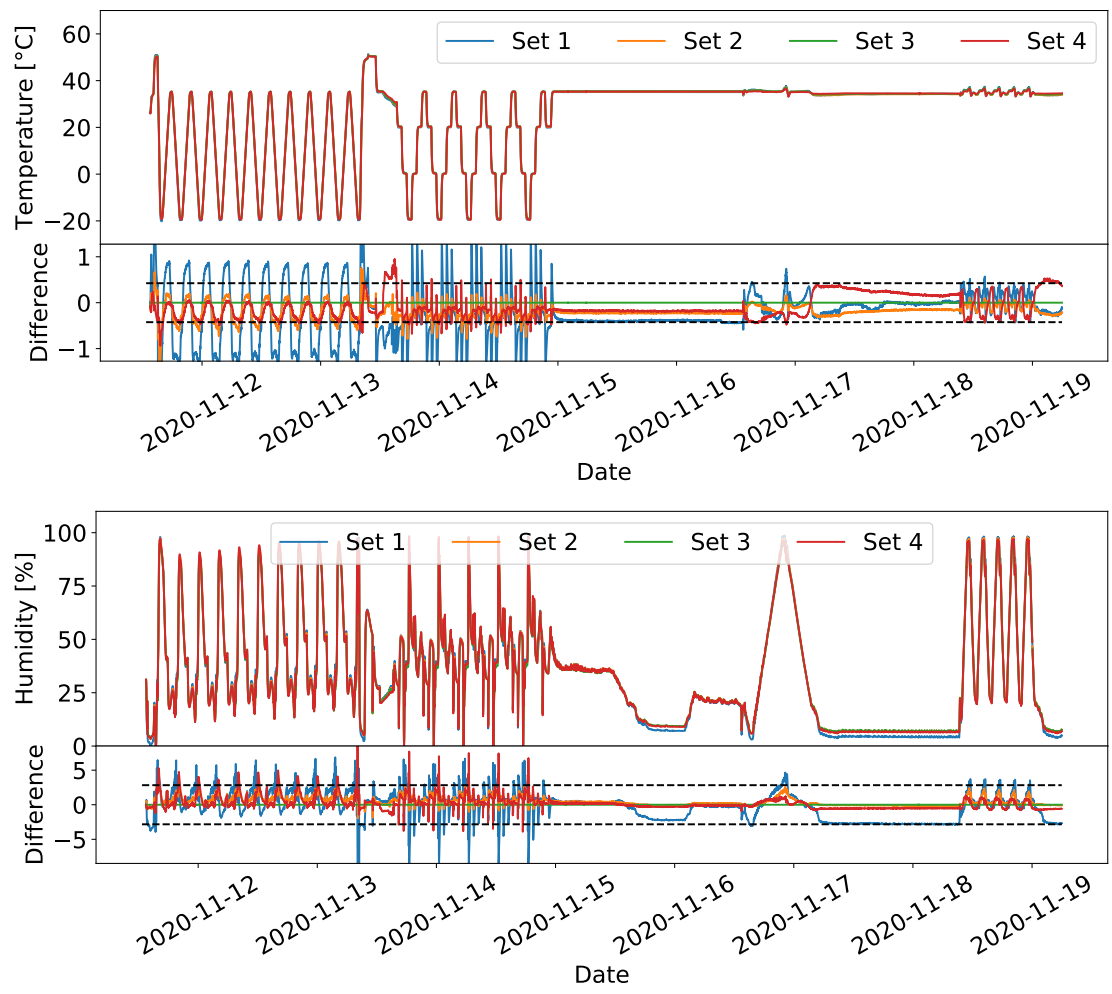

Figure 6: Results of climate chamber test. Black dashed lines in difference plots mark declared accuracy.

- Low humidity test and humidity cycling $5 \%$ to $100 \%$

Results are shown in Fig. 6. It is visible that there is a noticeable lag of measured temperatures due to the temperature distribution inside the chamber and relatively fast changes. There is a considerably larger deviation manifested by the set 1 , suggesting larger thermal capacity. Still, the difference is within $\pm 1{ }^{\circ} \mathrm{C}$, hence acceptable.

The reported humidity is similar among all sets with the set 1 reporting lower values during 
the low humidity test, though within the declared accuracy.

\section{Summary and outlook}

Four AEROSITE stations were prepared and tested to be deployed on SWGO candidate sites. Stations are being shipped and will be assembled on sites by local institutions personnel.

Cross-calibration campaign confirmed the manufacturer declared accuracy of temperature, humidity, pressure and solar irradiation. Anemometers were confirmed to reliably provide comparable data. Remaining sensors were tested in laboratory and compared with satisfactory result.

AEROSITEs will collect data on candidate sites for the period of at least two years starting ca. at the end of summer 2021. Data will be continuously evaluated and will serve as an input for the site selection process, which is aimed for conclusion at the end of the R\&D phase, late in 2023.

\section{Acknowledgements}

The work is supported by project LTT20002 of MŠMT, Czech Republic.

\section{References}

[1] A. Abeysekara, J. Aguilar, S. Aguilar, R. Alfaro, E. Almaraz, C. Álvarez et al., On the sensitivity of the HAWC observatory to gamma-ray bursts, Astroparticle Physics $\mathbf{3 5}$ (2012) 641.

[2] B. Bartoli, P. Bernardini, X.J. Bi, Z. Cao, S. Catalanotti, S.Z. Chen et al., Search for gamma-ray bursts with the ARGO-YBJ detector in shower mode, The Astrophysical Journal 842 (2017) 31.

[3] X. Bai, B.Y. Bi, X.J. Bi, Z. Cao, S.Z. Chen, Y. Chen et al., The large high altitude air shower observatory (LHAASO) science white paper, 2019.

[4] J. Hinton, The southern wide-field gamma-ray observatory: Status and prospects, in PoS ICRC 2021 ( $t b c$ ), vol. 395, (Berlin, DE), 2021.

[5] Reinhardt System und Messelectronic GmbHn, https://www.reinhardt-testsystem.de/english/climate_sensors.php, June, 2021.

[6] C. Fruck, M. Gaug, J.-P. Ernenwein, D. Mandát, T. Schweizer, D. Häfner et al., Instrumentation for comparing night sky quality and atmospheric conditions of CTA site candidates, Journal of Instrumentation 10 (2015) P04012.

[7] Gill Instruments Limited, http://gillinstruments.com/products/anemometer/windsonic.htm, June, 2021.

[8] Boltek Corp., https://www.boltek.com/efm-100-ss-06122020.pdf, June, 2021.

[9] M. Suchenek and T. Bulik, publication in preparation, .

[10] Balena, https://www.balena.io/fin/, June, 2021. 


\section{Full Authors List: SWGO Collaboration}

P. Abreu ${ }^{1}$, A. Albert ${ }^{2}$, E. O. Angüner ${ }^{3}$, C. Arcaro $^{4}$, L.H. Arnaldi ${ }^{5}$, J.C. Arteaga-Velázquez ${ }^{6}$, P. Assis ${ }^{1}$, A. Bakalová ${ }^{7}$, U. Barres de Almeida ${ }^{8}$, I. Batković ${ }^{4}$, J. Bellido ${ }^{9}$, E. Belmont-Moreno ${ }^{10}$, F. Bisconti ${ }^{11}$, A. Blanco ${ }^{1}$, M. Bohacova ${ }^{7}$, E. Bottacini ${ }^{4}$, T. Bretz ${ }^{12}$, C. Brisbois ${ }^{13}$, P. Brogueira ${ }^{1}$, A.M. Brown ${ }^{14}$, T. Bulik ${ }^{15}$, K. S. Caballero Mora ${ }^{16}$, S. M. Campos ${ }^{17}$ A. Chiavassa ${ }^{11}$, L. Chytka ${ }^{7}$, R. Conceição ${ }^{1}$, G. Consolati ${ }^{18}$, J. Cotzomi Paleta ${ }^{19}$, S. Dasso ${ }^{20}$, A. De Angelis ${ }^{4}$, C.R. De Bom ${ }^{8}$, E. de la Fuente ${ }^{21}$, V. de Souza ${ }^{22}$, D. Depaoli ${ }^{11}$, G. Di Sciascio ${ }^{23}$, C. O. Dib ${ }^{24}$, D. Dorner ${ }^{25}$, M. Doro ${ }^{4}$, M. Du Vernois ${ }^{26}$, T. Erginn $^{27}$, K. L. Fan ${ }^{13}$, N. Fraija ${ }^{8}$, S. Funk $^{28}$, J. I. García ${ }^{17}$, J. A. García-González ${ }^{29}$, S. T. García Roca ${ }^{9}$, G. Giacinti ${ }^{30}$, H. Goksu ${ }^{30}$, B. S. González ${ }^{1}$, F. Guarino ${ }^{31}$, A. Guillén ${ }^{32}$,

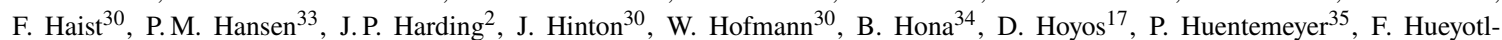
Zahuantitla $^{16}$ A. Insolia ${ }^{36}$, P. Janecek ${ }^{7}$, V. Joshi ${ }^{28}$, B. Khelifi ${ }^{37}$, S. Kunwar ${ }^{30}$, G. La Mura ${ }^{1}$, J. Lapington ${ }^{38}$, M. R. Laspiur ${ }^{17}$, F. Leit1 ${ }^{28}$, F. Longo ${ }^{39}$, L. Lopes ${ }^{1}$, R. Lopez-Coto ${ }^{4}$, D. Mandat ${ }^{7}$, A. G. Mariazzi ${ }^{33}$, M. Mariotti ${ }^{4}$, A. Marques Moraes ${ }^{8}$, J. MartínezCastro $^{40}$, H. Martínez-Huerta ${ }^{41}$, S. May ${ }^{42}$, D. G. Melo ${ }^{43}$, L. F. Mendes ${ }^{1}$, L. M. Mendes ${ }^{1}$, T. Mineeva ${ }^{24}$, A. Mitchell ${ }^{44}$, S. Mohan ${ }^{35}$, O. G. Morales Olivares ${ }^{16}$, E. Moreno-Barbosa ${ }^{19}$, L. Nellen ${ }^{45}$, V. Novotny ${ }^{7}$, L. Olivera-Nieto ${ }^{30}$, E. Orlando ${ }^{39}$, M. Pech $^{7}$, A. Pichel $^{20}$, M. Pimenta ${ }^{1}$, M. Portes de Albuquerque ${ }^{8}$, E. Prandini ${ }^{4}$, M. S. Rado Cuchills ${ }^{9}$, A. Reisenegger ${ }^{46}$, B. Reville ${ }^{30}$, C. D. Rho ${ }^{47}$, A. C. Rovero ${ }^{20}$ E. Ruiz-Velasco ${ }^{30}$, G. A. Salazar ${ }^{17}$, A. Sandoval ${ }^{10}$, M. Santander ${ }^{42}$, H. Schoorlemmer ${ }^{30}$, F. Schüssler ${ }^{48}$, V. H. Serrano ${ }^{17}$, R. C. Shellard ${ }^{8}$, A. Sinha ${ }^{49}$, A. J. Smith ${ }^{13}$, P. Surajbali ${ }^{30}$, B. Tomé ${ }^{1}$, I. Torres Aguilar ${ }^{50}$, C. van Eldik ${ }^{28}$, I. D. Vergara-Quispe ${ }^{33}$, A. Viana ${ }^{22}$, J. Vícha ${ }^{7}$, C. F. Vigorito ${ }^{11}$, X. Wang ${ }^{35}$, F. Werner ${ }^{30}$, R. White ${ }^{30}$, M. A. Zamalloa Jara ${ }^{9}$

${ }^{1}$ Laboratório de Instrumentação e Física Experimental de Partículas (LIP), Av. Prof. Gama Pinto 2, 1649-003 Lisboa, Portugal

${ }^{2}$ Physics Division, Los Alamos National Laboratory, P.O. Box 1663, Los Alamos, NM 87545, United States

${ }^{3}$ Aix Marseille Univ, CNRS/IN2P3, CPPM, 163 avenue de Luminy - Case 902, 13288 Marseille cedex 09, France

${ }^{4}$ University of Padova, Department of Physics and Astronomy \& INFN Padova, Via Marzolo 8 - 35131 Padova, Italy

${ }^{5}$ Centro Atómico Bariloche, Comisión Nacional de Energía Atómica, S. C. de Bariloche (8400), RN, Argentina

${ }^{6}$ Universidad Michoacana de San Nicolás de Hidalgo, Calle de Santiago Tapia 403, Centro, 58000 Morelia, Mich., México

${ }^{7}$ FZU, Institute of Physics of the Czech Academy of Sciences, Na Slovance 1999/2, 18200 Praha 8, Czech Republic

${ }^{8}$ Centro Brasileiro de Pesquisas Físicas, R. Dr. Xavier Sigaud, 150 - Rio de Janeiro - RJ, 22290-180, Brazil

${ }^{9}$ Academic Department of Physics - Faculty of Sciences - Universidad Nacional de San Antonio Abad del Cusco (UNSAAC), Av. de la Cultura, 733, Pabellón C-358, Cusco, Peru

${ }^{10}$ Instituto de Física, Universidad Nacional Autónoma de México, Sendero Bicipuma, C.U., Coyoacán, 04510 Ciudad de México, CDMX, México

${ }^{11}$ Dipartimento di Fisica, Università degli Studi di Torino, Via Pietro Giuria 1, 10125, Torino, Italy

${ }^{12}$ RWTH Aachen University, Physics Institute 3, Otto-Blumenthal-Straße, 52074 Aachen, Germany

${ }^{13}$ University of Maryland, College Park, MD 20742, United States

${ }^{14}$ Durham University, Stockton Road, Durham, DH1 3LE, United Kingdom

${ }^{15}$ Astronomical Observatory, University of Warsaw, Aleje Ujazdowskie 4, 00478 Warsaw, Poland

${ }^{16}$ Facultad de Ciencias en Física y Matemáticas UNACH, Boulevard Belisario Domínguez, Km. 1081, Sin Número, Terán, Tuxtla Gutiérrez, Chiapas, México

${ }^{17}$ Facultad de Ciencias Exactas, Universidad Nacional de Salta, Avda. Bolivia No 5150, (4400) Salta Capital, Argentina

${ }^{18}$ Department of Aerospace Science and Technology, Politecnico di Milano, Via Privata Giuseppe La Masa, 34, 20156 Milano MI, Italy

${ }^{19}$ Facultad de Ciencias Físico Matemáticas, Benemérita Universidad Autónoma de Puebla, C.P. 72592, México

${ }^{20}$ Instituto de Astronomia y Fisica del Espacio (IAFE, CONICET-UBA), Casilla de Correo 67 - Suc. 28 (C1428ZAA), Ciudad Autónoma de Buenos Aires, Argentina

${ }^{21}$ Universidad de Guadalajara, Blvd. Gral. Marcelino García Barragán 1421, Olímpica, 44430 Guadalajara, Jal., México

${ }^{22}$ Instituto de Física de São Carlos, Universidade de São Paulo, Avenida Trabalhador São-carlense, no 400, Parque Arnold Schimidt CEP 13566-590, São Carlos - São Paulo - Brasil

${ }^{23}$ INFN - Roma Tor Vergata and INAF-IAPS, Via del Fosso del Cavaliere, 100, 00133 Roma RM, Italy

${ }^{24}$ Dept. of Physics and CCTVal, Universidad Tecnica Federico Santa Maria, Avenida España 1680, Valparaíso, Chile

${ }^{25}$ Universität Würzburg, Institut für Theoretische Physik und Astrophysik, Emil-Fischer-Str. 31, 97074 Würzburg, Germany

${ }^{26}$ Department of Physics, and the Wisconsin IceCube Particle Astrophysics Center (WIPAC), University of Wisconsin, 222 West Washington Ave., Suite 500, Madison, WI 53703, United States

27 TUBITAK Space Technologies Research Institute, ODTU Campus, 06800, Ankara, Turkey

${ }^{28}$ Friedrich-Alexander-Universität Erlangen-Nürnberg, Erlangen Centre for Astroparticle Physics, Erwin-Rommel-Str. 1, D 91058 Erlangen, Germany

${ }^{29}$ Tecnologico de Monterrey, Escuela de Ingeniería y Ciencias, Ave. Eugenio Garza Sada 2501, Monterrey, N.L., 64849, México

${ }^{30}$ Max-Planck-Institut für Kernphysik, P.O. Box 103980, D 69029 Heidelberg, Germany

${ }^{31}$ Università di Napoli "Federico II", Dipartimento di Fisica "Ettore Pancini”, and INFN Napoli, Complesso Universitario di Monte Sant'Angelo - Via Cinthia, 21 - 80126 - Napoli, Italy

${ }^{32}$ University of Granada, Campus Universitario de Cartuja, Calle Prof. Vicente Callao, 3, 18011 Granada, Spain 
${ }^{33}$ IFLP, Universidad Nacional de La Plata and CONICET, Diagonal 113, Casco Urbano, B1900 La Plata, Provincia de Buenos Aires, Argentina

34 University of Utah, 201 Presidents' Cir, Salt Lake City, UT 84112, United States

${ }^{35}$ Michigan Technological University, 1400 Townsend Drive, Houghton, MI 49931, United States

${ }^{36}$ Dipartimento di Fisica e Astronomia "E. Majorana", Catania University and INFN, Catania, Italy

${ }^{37}$ APC-IN2P3/CNRS, Université de Paris, Bâtiment Condorcet, 10 rue A.Domon et Léonie Duquet, 75205 PARIS CEDEX 13, France

${ }^{38}$ University of Leicester, University Road, Leicester LE1 7RH, United Kingdom

${ }^{39}$ Department of Physics, University of Trieste and INFN Trieste, via Valerio 2, I-34127, Trieste, Italy

${ }^{40}$ Centro de Investigación en Computación, Instituto Politécnico Nacional, Av. Juan de Dios Bátiz S/N, Nueva Industrial Vallejo, Gustavo A. Madero, 07738 Ciudad de México, CDMX, México

${ }^{41}$ Department of Physics and Mathematics, Universidad de Monterrey, Av. Morones Prieto 4500, San Pedro Garza García 66238, N.L., México

42 Department of Physics and Astronomy, University of Alabama, Gallalee Hall, Tuscaloosa, AL 35401, United States

${ }^{43}$ Instituto de Tecnologías en Detección y Astropartículas (CNEA-CONICET-UNSAM), Av. Gral Paz 1499 - San Martín - Pcia. de Buenos Aires, Argentina

${ }^{44}$ Department of Physics, ETH Zurich, CH-8093 Zurich, Switzerland

${ }^{45}$ Instituto de Ciencias Nucleares, Universidad Nacional Autónoma de México (ICN-UNAM), Cto. Exterior S/N, C.U., Coyoacán, 04510 Ciudad de México, CDMX, México

${ }^{46}$ Departamento de Física, Facultad de Ciencias Básicas, Universidad Metropolitana de Ciencias de la Educación, Av. José Pedro Alessandri 774, Ñuñoa, Santiago, Chile

${ }^{47}$ Department of Physics, University of Seoul, 163 Seoulsiripdaero, Dongdaemun-gu, Seoul 02504, Republic of Korea

${ }^{48}$ Institut de recherche sur les lois fondamentales de l'Univers (IRFU), CEA, Université Paris-Saclay, F-91191 Gif-sur-Yvette, France

${ }^{49}$ Laboratoire Univers et Particules de Montpellier, CNRS, Université de Montpelleir, F-34090 Montpellier, France

${ }^{50}$ Instituto Nacional de Astrofísica, Óptica y Electrónica (INAOE), Luis Enrique Erro 1, Puebla, México 